\title{
Método de Ecuaciones Estructurales Econométricas para el Análisis de Gestión en Ciencia, Tecnología e Innovación
}

\author{
María I. Rojas ${ }^{(1) \star}$ y Ruby L. Espejo(2) \\ (1) Escuela de Economía, Univ. Pedagógica y Tecnológica de Colombia (UPTC), Tunja, Boyacá, Colombia. \\ (e-mail: maria.rojas03@uptc.edu.co). \\ (2) Facultad de Estudios a Distancia, Escuela de Ciencias Humanísticas y Educación (UPTC), Tunja, Boyacá, \\ Colombia. (e-mail: ruby.espejo@uptc.edu.co).
}

${ }^{*}$ Autor a quien se debe enviar la correspondencia

Recibido Ene. 2, 2018; Aceptado Mar. 28, 2018; Versión final May. 25, 2018, Publicado Oct. 2018

\begin{abstract}
Resumen
El objetivo del estudio es proponer un método para analizar en conjunto la gestión en ciencia, tecnología e innovación de las universidades, gobierno y empresa privada. La metodología integra un sistema de ecuaciones econométricas, de acuerdo con conceptos de la literatura económica y administrativa, observando indicadores de línea base. Los hallazgos, en particular para Boyacá-Colombia, muestran integración tripartita en investigación entre los grupos activos de investigación para la producción científica, los proyectos Colciencias como retornos de inversión en actividades científicas, y la oferta de programas de las universidades para producir patentes. Estos resultados pueden ser asequibles para rediseñar políticas de investigación y desarrollo.
\end{abstract}

Palabras clave: indicadores de ciencia, tecnología e innovación; producción científica; análisis estructurales econométricos

\section{Method of Econometric Structural Equations for Management Analysis in Science, Technology and Innovation}

\begin{abstract}
The purpose of the study is to propose a method to analyze altogether the management in science technology and innovation of the universities, government and private sector. The methodology employed in this study integrates an econometric equation system, according with economic and administrative literature concepts; observing baseline indicators. The findings, particularly for Boyacá-Colombia, show tripartite integration in research among the active research groups for scientific production, the Colciencias projects as investment returns in scientific activities and the offer of university programs to produce patents. These results are of importance for the design of public policies of research and development.
\end{abstract}

Keywords: science, technology and innovation indicators; scientific production; structural econometric analysis 


\section{INTRODUCCIÓN}

Parte importante de las actividades relacionadas con la gestión administrativa, académica y científica de la investigación, que realizan las instituciones de educación superior, las entidades gubernamentales y algunas organizaciones privadas dedicadas a la investigación científica es la obtención de información primaria para la construcción de indicadores sobre ciencia, tecnología e innovación (CTel), y la evaluación del quehacer, en investigación, de los actores relevantes para el desarrollo y la productividad de una región; razón por la cual es necesario contar con herramientas que permitan cumplir con dichas dinámicas. En el marco de las dinámicas académicas que se concentran en CTel, existe una alta heterogeneidad (Luintel y Mosahid, 2009) de trabajos, presentados a través de libros, artículos y otros medios (Hitt et al, 1998) que frecuentemente visualizan y evalúan la investigación, atendiendo con gran interés a la ciencia (Frietsch et al., 2009) y a la innovación (Mairesse y Mohnen, 2010; Becheikh et al, 2006; Wolfgang y Jürgen, 2002; Ahuja, 2000; Crepon et al, 1998), y poniendo especial atención al rol del potencial humano (Espejo, 2015 - 2018). Además, algunas estrategias para aumentar la innovación (Álvarez y Bernal (2017) se concentran más en lo tecnológico, en función de la eficiencia de transferencias y porcentajes de regalías (Link y Siegel, 2005; Hernández et al, 2014; Valdés et al, 2002). En particular para este propósito, se considera clave la capacidad de gestionar el conocimiento (Mateus y Giraldo, 2012) como motor de la innovación, al aplicar estrategias de visibilidad, con el fin de interrelacionar estructuras y modelos complejos (Pires y Machado, 2006; Cruz, 2004).

Asimismo, se encuentra gran cantidad de contenidos investigativos, que integran la CTel (Palomo et al., 2007), donde juegan un papel importante las revistas. Información Tecnológica, (2014), entre otras, muestra la recepción y la publicación de dichos contenidos, dinamizada a través de la demanda. Sin embargo, se percibe una tensión (Neumann y Weigand, 2004; Callón, (1999) entre cómo superar las necesidades de financiación universitaria y lo contraproducente de la presencia del poder público. En este sentido, no se puede desconocer el panorama internacional y nacional de la CTel, en el que Colombia avanza (Rodríguez et al., 2001), así como lo hace América Latina; en tal contexto, es importante tener en cuenta la medición de los indicadores en CTel, con parámetros y metodologías de los manuales de la Organización para la Cooperación y el Desarrollo Económico (OCDE), los de Frascati, Oslo y los de Bogotá (Sancho, 2002). El Departamento Administrativo Nacional de Estadística (DANE), en Colombia, también publica una serie de indicadores a nivel micro con metodologías similares. Por su parte, las entidades encargadas de la CTel para el departamento de Boyacá aúnan esfuerzos para calcular indicadores base en CTel, para ser publicados periódicamente, pero sin profundizar en la medición de su gestión, tan necesaria para evaluar y plantear políticas públicas y privadas que puedan alcanzar los objetivos que persigue el conocimiento. El poder contar con aportes explícitos en los trabajos de Echeverri, et al. (2018) y Hernández, et al. (2017) en relación con la gestión de las organizaciones, y el poder concretar prácticas de incidencia creativa, impulsa y contextualiza el interés de lo que sucede en la CTel y, a la vez, deja evidencia sobre los niveles de innovación tecnológica y su radio de acción dentro de las dinámicas enmarcadas en el desarrollo organizacional. Así mismo, Pinto, et al. (2017) encuentra algunos aspectos que anteceden al éxito organizacional, y que están relacionados con los proyectos y las personas, destacando la comunicación efectiva, el uso de sistemas y la participación personal.

Los análisis correlacionales evidencian que el desarrollo de la gestión del conocimiento, en cada empresa, y de acuerdo con el ciclo de vida, las tecnologías digitales, la cultura organizacional, los procesos estratégicos y misionales se corresponde con el grado de apoyo a las competencias personales y relacionales de sus miembros (López, et al., 2015); esto se asemeja a lo que Marulanda, et al. (2013) develan acerca de la correlación hallada entre las redes de tecnologías de la información y la comunicación y el tipo de prácticas para innovar encontradas en el eje cafetero en Colombia. Sin embargo, de acuerdo con los informes puntuales sobre innovación e inversión en investigación y desarrollo en América Latina y El Caribe (ALC), las estadísticas evidencian bajos niveles de rendimiento en innovación, en comparación con países desarrollados, como los de la OCDE y países emergentes como China, especialmente en sectores de la electrónica. La debilidad se presenta en aquellos campos tecnológicos que se han identificado con los nuevos paradigmas en la biotecnología y las tecnologías de la información y las comunicaciones (Guzmán et al., 2012).

En relación con el desarrollo de la industria innovadora, en estudios recientes realizados por el DANE y el Observatorio de Ciencia y Tecnología para Boyacá, respectivamente para el país y el departamento, se presentan cifras sobre las empresas innovadoras en Colombia y en particular para Boyacá, en los períodos 2009-2010 y 2011-2012. Las cifras muestran una disminución, entre el primero y segundo período, del 60,8\% de empresas innovadoras (entendiendo aquí innovación en sentido estricto) en el país, mientras que se registra solamente una (1) empresa innovadora en Boyacá, en ambos períodos. Respecto a las empresas innovadoras (esta vez innovación en un sentido amplio), la caída es de un 38,2\%, pasando de 51 a 30 empresas, de uno a otro período, mientras que Boyacá registra treinta (30) empresas en ambos periodos. En relación con las empresas potencialmente innovadoras, se presenta, para Colombia, una disminución de $3,7 \%$, mientras que para Boyacá la disminución es del $50 \%$, al pasar de 8 a 4 empresas, de uno a otro período. 
En cuanto al número de empresas no innovadoras, se da, en el país, un incremento del $24,4 \%$, el equivalente a 1.105 empresas, entre los dos períodos; igual ocurre en Boyacá, que pasa de registrar 35 a 46 empresas no innovadoras (DANE, 2013; UPTC, 2014). Las anteriores cifras, para Colombia y Boyacá, no son halagadoras en relación con la innovación; otro ejemplo de cifras desalentadoras se encuentra en el área de la tecnología médica, estudiada a través de las solicitudes de patentes; estas, representaron un 39\% del total para Colombia entre 1999 y 2013, y solo fueron utilizadas en algunos sectores donde los productos son difíciles de imitar (Langebaek y Escobar, 2007); mientras que Boyacá, en el periodo de estudio 2003 a 2015 , registra entre 0 y 2 patentes de invención, y entre 0 y 4 modelos de utilidad por año (UPTC, 2015).

Respecto a los gastos en Investigación y Desarrollo (I+D) -los cuales son gastos corrientes y de capital público y privado, donde se incluyen los conocimientos sobre la humanidad, la cultura y la sociedad, y el uso de estos para nuevas aplicaciones, y que abarcan la investigación básica, la investigación aplicada y el desarrollo experimental- fueron, en 2007, en promedio del 2,3\% del PIB, para las economías avanzadas (Quiroga et al., 2014); mientras que en ALC fueron del 0,67\%. Los registros en Colombia fueron del 0,20\%, mientras que para Boyacá solo representan el 0,023\%, muy por debajo de ALC y de las economías avanzadas y emergentes (UPTC, 2014).

Junto a estos datos, se revela la problemática que Colombia comparte con algunas regiones del mundo, por ejemplo, el hecho de que se cuenta con una baja densidad de investigadores por cantidad de población, y con una escasa inversión en ciencia y tecnología. No obstante, la conciencia respecto a la importancia del capital intelectual se ha venido extendiendo desde su génesis en la economía, con enfoques y modelos que se interpretan según su utilidad ética y visión, donde interactúan componentes del capital humano, estructural y relacional, en lo administrativo y lo contable; todo ello, explicado por la riqueza de los intangibles en las organizaciones (Bueno et al., 2008). En el sentido microeconómico, Mankiw (2005) pretende averiguar si, desde el punto de vista de la sociedad, las empresas maximizadoras del beneficio tienden a realizar demasiada o poca investigación. Se trata entonces de modelos de crecimiento endógeno, en los que la investigación toma parte en las decisiones de las empresas, en tanto, a su vez, dichas decisiones afectan a la innovación. Por lo anterior, el presente estudio propone una metodología para analizar la gestión en CTel en Boyacá, Colombia, teniendo en cuenta la literatura económica y administrativa referida a la investigación científica, y el fomento a la tecnología y a la innovación en los sectores académico, empresarial y gubernamental, con el fin de generar nuevos datos de referencia para la toma de decisiones y el diseño de políticas públicas que sirvan como recurso estratégico para el desarrollo de una región.

\section{PANORAMA ORGANIZACIONAL Y ECONÓMICO DE LA CTeI}

Un panorama de la ciencia, la tecnología y la innovación en los países en vía de desarrollo puede obtenerse a través del análisis de la acumulación del capital intelectual en las organizaciones, como elemento clave, al combinar los recursos físicos y humanos para promover la innovación y la tecnología (Secundo et al., 2010). Autores como Martínez (2003), Bueno et al., (2008), Diez et al., (2010) y Santos et al. (2010) aseguran que al reproducir el capital relacional y estructural, denominado capital intelectual, se desarrolla la productividad, la competitividad y el crecimiento económico de una región.

El capital estructural, representado en el conocimiento propio de las instituciones, surge en la medida que lo poseen las personas y los equipos; está compuesto por el capital organizativo y el capital tecnológico (Bueno et al., 2008). Al interactuar las acciones de las personas, se responde a los incentivos de los mercados, al cambio tecnológico, de forma endógena y no exógena, como se plantea en Romer (1990). En este sentido, en el contexto universitario, el capital organizativo se relaciona con elementos de la cultura, el aprendizaje y los procesos organizativos; el capital tecnológico, con los esfuerzos en investigación y desarrollo, la dotación tecnológica y la propiedad intelectual e industrial y la infraestructura básica, así como con los recursos generados por los datos empíricos manifiestos en bibliografías, compilaciones documentales, bases de datos, sistemas de gestión, patentes, nuevas tecnologías y material de laboratorios.

El capital relacional está compuesto por el capital de negocio y el capital social. El primero, relacionado con los principales agentes vinculados con la investigación y los clientes, los proveedores, los accionistas, las instituciones reguladoras e inversoras, aliados y competidores; y en lo social, se relaciona con el valor que una institución ha logrado desarrollar por medio del cumplimiento de la responsabilidad en lo político, lo económico y lo ambiental, y se expresa en términos del nivel de integración, el compromiso, la cooperación y la responsabilidad social, con lo cual se logra potencializar nuevos investigadores con apropiación del conocimiento y valor de marca (Bueno et al., 2008).

Ahora bien, en el contexto económico, desde los análisis y los aportes de Schumpeter (1997), el fomento a la innovación y a la tecnología presenta cambios cualitativos que implican alteraciones en la técnica de "producir" 
en las organizaciones y, en consecuencia, en el crecimiento y la evolución continua del espíritu de las empresas. Desde la perspectiva de Mankiw (2005) aborda la investigación y el desarrollo, en tres hechos evidentes: 1) aunque los conocimientos son un bien público al que todo el mundo puede acceder libremente, gran parte de las empresas tienen como fin el obtener beneficios; 2) la investigación es rentable por que las innovaciones dan a las empresas un monopolio temporal, bien a causa del sistema de patentes, o bien porque es una ventaja ser la primera en aparecer en el mercado con un nuevo producto; y 3) cuando una empresa innova, otras se basan en esa innovación para producir la siguiente generación de innovaciones.

Estos hechos no se relacionan con modelos de crecimiento macroeconómico, sino que siempre que una empresa crea nueva tecnología, mejora la situación de otras al darles unos conocimientos en los que pueden basar sus futuras investigaciones, o puede suceder que cuando una empresa invierte en investigación también puede empeorar la situación de otras, simplemente por ser la primera en descubrir una tecnología que otra habría inventado, surgiendo el efecto pisar las ideas. Esta externalidad es importante y tiene como consecuencia que el rendimiento social de la investigación sea grande, a menudo, superior al $50 \%$ por año. Generalmente, la tasa de rendimiento es superior a, o aproximadamente del 8\% anual, en países desarrollados. Aun cuando se compara con la inversión en capital físico, dicho rendimiento justifica la concesión de subvenciones públicas a la investigación.

Lo anterior indica que, desde el punto de vista económico del cambio tecnológico, el conocimiento es un activo productivo moderno que se almacena en un conjunto de conocimientos prescriptivos y se retroalimenta del conocimiento proposicional, y este, a su vez, de la investigación científica. En consecuencia, el saber integrado del conocimiento y la experiencia personal proporciona elementos claves para medir el capital humano de una forma implícita o tácita. Desde Becker (1975), Schultz (1981) y Lucas (1998), este capital en estado estacionario se asume como el motor del crecimiento económico, factor de producción que afecta a otros factores, además de la productividad marginal y de las externalidades positivas se tiene en cuenta que es la eficiencia asimilada en el sistema educativo (Fagerberg et al., 2010), similar a lo que Romer (1990) define como efecto acumulado de la educación y la experiencia, por lo que se propone tratar a la educación como una inversión en el hombre y, en consecuencia, como una forma de capital.

Se entiende entonces que los procesos de formación del capital humano en las universidades conducen a crear capital estructural, si se tiene en cuenta que en este último interviene prioritariamente la creatividad y el espíritu crítico, creando innovación tecnológica y científica; y el capital estructural es desarrollado por el docente-investigador, los directivos, los grupos y semilleros de investigación, los jóvenes investigadores, los doctores, los magísteres y los asesores de investigación adscritos a los centros investigativos en los que confluyen la formación y el aprendizaje. Es en este contexto que se ofrece el caso para el análisis de la gestión en CTel, para Boyacá, Colombia, clasificando los indicadores según el tipo de capital intelectual: humano, estructural o relacional, tomado del Boletín Línea Base de indicadores CTI Boyacá, UPTC., 2014 y 2015.

\section{METODOLOGÍA Y MEDICIÓN}

De acuerdo con la propuesta del presente trabajo, el análisis se centra en explicar la relación entre dos o más variables; va más allá de la descripción de los conceptos, por lo que define un estudio de tipo explicativo (Hernández et al., 2006). Se aborda en forma cuantitativa con diseño deductivo, apoyado en la teoría económica, estadística y econométrica. En la medición es necesario tener presente que desde el punto de vista econométrico (en Gujarati, 2010) se plantea que la decisión de invertir en I+D y su compensación definitiva en términos de mayor productividad implica un considerable rezago en la realidad; tal es el caso, entre la inversión de los fondos y el momento en el cual los inventos realmente empiezan a aparecer; según Griliches (1998), citado por Gujarati, existe un rezago entre la invención de una idea o mecanismo y su desarrollo, hasta llegar a la etapa en que sea comercialmente aplicable.

Con base en la teoría de Romer (1994; citado por Tejedor et al., 2018), a) Hay muchas firmas en una economía de mercado, no existe un modelo donde se tienda a concentrar toda la producción en manos de un monopolio para toda la economía. b) Los descubrimientos se diferencian de otros insumos en el sentido de que muchas personas pueden utilizarlos al mismo tiempo: las principales ideas —que han permitido comprender algunos principios básicos y comúnmente aplicados - tienen la propiedad de que ofrecen información que es tecnológicamente posible para todos los agentes. c) Es posible replicar las actividades físicas, esto implica que la función de producción agregada en un mercado competitivo debe caracterizarse por la homogeneidad grado uno en todos sus insumos convencionales (rivales), luego la producción científica se representa con la función $Y=A^{*} F(K, H, L)$, entonces, al duplicar todos los insumos de $K, H, L$, se debería permitir duplicar el producto. 
Además, el avance tecnológico viene de las cosas que la gente hace, el cambio tecnológico no está en función de un tiempo progresivo a nivel calendario y, se debe desligar el nexo entre la endogeneidad y la aleatoriedad. Se propone que la tasa global de descubrimientos es endógena y está determinada por las cosas que hace la gente. Las personas y las empresas tienen poder de mercado y ganan rentas de monopolio con los descubrimientos. Luego, la gente y las firmas tienen cierto control sobre la información producida por la mayoría de los descubrimientos. Una empresa puede controlar el acceso a un descubrimiento, puede cobrar un precio que es superior a cero, así que gana los beneficios del monopolio porque la información no tiene costo de oportunidad. Ahora, el modelo que propone Romer (1990; citado por Tejedor et al., 2018), basado en la función de producción de Cobb-Douglas, es diferente de la función de producción tradicional, ya que posee otros tipos de bienes de capital que no son sustitutos perfectos entre sí. Este modelo está dado por la ecuación (1):

$$
Y\left(H_{y}, L, X\right)=H_{y}^{\alpha} L^{\beta} \sum_{i=1}^{\infty} x_{i}^{1-\alpha-\beta}
$$

La ecuación (1) presenta el producto final representado por $Y$, el cual está en función de los insumos capital humano $\mathrm{H} \gamma$, de la mano de obra física $(L)$ y la tecnología de producción $(X)$ que desagrega el capital en un número infinito de tipos diferentes de bienes duraderos: se indican por un entero $\mathrm{i}$, solo los insumos que ya se han inventado y diseñado, y están disponibles para usarse. El capital humano $\mathrm{H}$ mide el efecto acumulado de la educación y la experiencia, corresponde al conocimiento, el cual es un bien rival; la mano de obra $L$ son los servicios de trabajo expresados en el número de personas aptas para realizarlos; y el capital físico $X$ define implícitamente el capital $\mathrm{K}$ proporcional a la suma de todos los tipos de bienes de capital diferentes de la economía, sustitutos perfectos cercanos, pares complementarios y pares intermedios.

En el modelo se separa al componente rival del conocimiento $\mathrm{H}$, del componente no rival del conocimiento aplicado a un diseño tecnológico $\mathrm{A}$, el cual puede crecer ilimitadamente, lo cual ocurre únicamente en ausencia de derechos de propiedad para el uso de los nuevos conocimientos dentro del proceso de producción. A es el componente tecnológico no rival, y corresponde a cada nueva unidad de conocimiento aplicada a un diseño para un nuevo bien. $X=$ [xi] (desde $i=1$ hasta $\infty$ ) es la lista de los insumos usados por una empresa que obtiene un producto final. Hay cierto valor de $A$ tal que $X i=0$ para todo $i \geq A$. Luego $A$ cambia a medida que se inventan nuevos bienes de productos duraderos. El producto final se describe como una función estacionaria de todas las listas de insumos concebibles. Además, al sumar el número de personas ocupadas en investigación, el acervo agregado de los diseños evolucionará de acuerdo con la ecuación (2), donde HA es el capital humano total empleado en la investigación:

$$
A=\delta H_{A} A
$$

Todos los investigadores pueden aprovecharse de $A$ al mismo tiempo. Por lo tanto, el producto del investigador j es $\delta H_{j} A$. Romer (1990) explica que el nuevo conocimiento es apropiable libremente en el proceso de producción porque al ser copiados los nuevos diseños, se permite fabricar productos nuevos, lo cual aumenta el acervo de conocimiento e incrementa la productividad del capital humano en el sector de investigación, aunque se pueden presentar conflictos cuando un nuevo diseño es copiado. El efecto de no poseer derechos de propiedad sobre el conocimiento hace que su acceso por parte del sector I+D genere mayor crecimiento en $\mathrm{A}$ y, por ello, en la productividad del capital humano: el crecimiento en $A$ incrementa por sí mismo la productividad del capital humano en el sector de investigación; el equilibrio se basa aquí en el supuesto de que quienquiera que se ocupe de la investigación tiene libre acceso al acervo total de conocimientos (Tejedor et al., 2018).

En el otro escenario de Mankiw (2005), se representa la función de producción para incorporar el progreso tecnológico y la eficiencia del trabajo, dada por la ecuación (3):

$$
Y=f\left(K, L^{*} E\right)
$$

Donde $E$ es una nueva variable (abstracta) Ilamada eficiencia del trabajo; esta pretende reflejar los conocimientos de la sociedad sobre los métodos de producción: a medida que mejora la tecnología existente, la eficiencia del trabajo aumenta. Por ejemplo, aumenta cuando mejora la educación o las cualificaciones de la población activa. $L^{\star} E$ mide el número de trabajadores efectivos; tiene en cuenta el número de trabajadores, $L, y$ la eficiencia de cada uno, $E$.

El supuesto sobre el progreso tecnológico es el que hace que la eficiencia del trabajo crezca, a una tasa constante $g$. Si $L$ crece a una tasa $n$, y la eficiencia de cada unidad de trabajo $E$, a la tasa $g$, entonces el número de trabajadores efectivos crecerá a una tasa $n+g$. La producción total $Y$, en las ecuaciones (1) y (3), 
se representa con el indicador Producción científica, que se expresa con el número de documentos publicados en WoS (Web of Science, plataforma web que recoge referencias de publicaciones científicas), registrados en Boyacá, como variable dependiente, siendo la publicación de documentos más representativa, dado que el número de publicaciones de SCOPUS y revistas indexadas en Publindex (bases de datos de publicaciones científicas) se encuentran traslapadas en WoS.

Como variable explicativa, el insumo Capital (K), en las ecuaciones (1) y (3), se refleja en la inversión en edificios y laboratorios destinados a la investigación, lo cual se recoge con la inversión en ACTI (actividades de CTel) para Boyacá (dada en millones de pesos constantes, con 2014 como año base). La inversión en I+D se encuentra inmersa en las actividades de investigación y desarrollo de las instituciones educativas y de las empresas. L, en las ecuaciones (1) y (3), representa el insumo trabajo, el cual se identifica por el indicador número de grupos de investigación avalados por instituciones registradas en Boyacá.

En la ecuación (3), E es una variable latente que se relaciona con el capital humano; se mide mediante el indicador Número total de graduados en las instituciones de educación, por los niveles: técnica profesional, tecnológica, pregrado universitario, especialización, maestría y doctorado. La acumulación del capital humano, puede ser el resultado de la asistencia a instituciones educativas, o del "aprender haciendo" (Arrow, 1962), así como de las horas dedicadas en los puestos de trabajo (Lucas, 1998; Romer, 1990). Finalmente, $L^{*} E$, en el contexto de este estudio, equivale al número de grupos activos multiplicado por el número de graduados. Con la teoría económica de Romer (1990) y Mankiw (2005), y la teoría del capital intelectual en las organizaciones, es posible visualizar la interacción que tiene la inversión en CTel, la gestión en capital relacional que logran las instituciones, y las actividades desarrolladas por su capital humano dedicado a crear capital estructural.

Atendiendo a las consideraciones expuestas, particularmente a las referidas a las interacciones entre los elementos del capital estructural, relacional y humano relacionado con la CTel de las instituciones que adelantan investigación científica, se plantea un modelo econométrico multiecuacional representado en las ecuaciones estructurales (4), (5), (6) y (7), cuyas variables y datos se identifican en la tabla 1.

\section{Variables}

Acti_B: inversión en actividades de ciencia, tecnología e innovación ( ${ }^{*} e n$ millones de pesos, de 2014); graduados: número de graduados por año en Boyacá, incluyendo los niveles de educación: técnica profesional, tecnológica, pregrado universitario, especialización, maestría y doctorado; becas: número de beneficiarios de becas para doctorado en Boyacá; ofer_program: número de programas por nivel académico y área OCDE por año, en Boyacá; producc_cie: producción científica en Boyacá. Muestra: número de publicaciones en WoS; grup_acti: número de grupos de investigación activos, según entidad territorial en Boyacá; patentes: número de concesiones de patentes a residentes en Boyacá; proyec_col: número de proyectos aprobados para financiamiento en Colciencias, en Boyacá.

Tabla 1: Variables y datos de los indicadores Línea Base de CTI para Boyacá - Boletines 2014 y 2015 - UPTC

\begin{tabular}{|c|c|c|c|c|c|c|c|c|}
\hline Año & Acti_B & Graduados & becas & ofer_program & producc_cie & grup_acti & patentes & proyec_colc \\
\hline 2003 & 6261 & 5208 & 4 & 194 & 11 & 69 & 0 & 2 \\
\hline 2004 & 5716 & 4215 & 6 & 253 & 16 & 87 & 0 & 0 \\
\hline 2005 & 7746 & 3605 & 5 & 250 & 13 & 108 & 0 & 5 \\
\hline 2006 & 6202 & 3593 & 8 & 272 & 11 & 129 & 0 & 3 \\
\hline 2007 & 6344 & 4856 & 4 & 704 & 43 & 145 & 0 & 2 \\
\hline 2008 & 6406 & 3807 & 8 & 772 & 62 & 150 & 1 & 3 \\
\hline 2009 & 7734 & 5005 & 2 & 872 & 76 & 148 & 1 & 2 \\
\hline 2010 & 10921 & 6972 & 7 & 928 & 159 & 139 & 2 & 3 \\
\hline 2011 & 7296 & 10004 & 18 & 905 & 182 & 142 & 1 & 3 \\
\hline 2012 & 17832 & 9009 & 33 & 975 & 241 & 150 & 0 & 3 \\
\hline 2013 & 28043 & 7042 & 7 & 1185 & 251 & 144 & 2 & 5 \\
\hline 2014 & 33723 & 7735 & 15 & 1090 & 272 & 126 & 1 & 6 \\
\hline
\end{tabular}

El modelo econométrico responde de forma estructural a la transformación logarítmica de una función de producción con tecnología tipo Coob-Douglas de la ecuación (3) a la ecuación (4). 


$$
\begin{aligned}
& \log (\text { ProducciónCientífica })=\beta_{0}+\beta_{1}{ }^{*} \log \left(\operatorname{Inv} v_{A C T I}\right)+\beta_{2}{ }^{*} \log \left(\operatorname{Grupos}_{\text {Activos }}{ }^{*} \text { Graduados }\right)+\varepsilon_{t 1} \\
& \left(\text { Acti }_{b}\right)=\beta_{4}+\beta_{5}{ }^{*}\left(\text { Proyec }_{\text {Colc }}\right)+\beta_{6} *(\text { Becas })+\varepsilon_{t 2} \\
& (\text { Patentes })=\beta_{7}+\beta_{8}{ }^{*}\left(\text { Graduados }^{*} \text { Grupo }_{\text {acti }}\right)+\beta_{9}{ }^{*}\left(\text { Oferta }_{\text {programa }}\right)+\varepsilon_{t 3}
\end{aligned}
$$

(Inst) : $\beta_{0}$ GraduadosGrup $_{\text {Activos }}$ Ofer $_{\text {Program }}$ Proyect $_{\text {colc }}$ Producc $_{\text {cie }}(-1)$

La ecuación (4) representa la producción científica en función de la inversión en Actividades de CTel para Boyacá (ACTI), así como la capacidad potencial para producir, esta última presentada como el producto entre el número de grupos de investigación activos por el número de graduados, denominado por Mankiw trabajo efectivo. Los grupos de investigación y los graduados son los encargados de fomentar el progreso tecnológico. De esta forma, están siendo estimados los efectos del capital humano en la producción científica. En la Ecuación (5) se estima el capital relacional; este representa los esfuerzos de la investigación en función de los proyectos conseguidos, dadas las relaciones sociales y la responsabilidad que se tiene con la comunidad. El gasto en investigación ( Acti $_{b}$ ), variable dependiente, representa la inversión en las actividades de CTel para Boyacá, en función de los proyectos avalados por Colciencias y las becas otorgadas.

La ecuación (6) permite identificar las acciones que se han llevado a cabo en investigación y desarrollo mediante las patentes, en función de la eficiencia o progreso tecnológico. Se supone que los graduados están inmersos en el mercado laboral, los grupos activos de investigación y la oferta de programas de las universidades, estimando así el capital estructural. La instrucción (7) se da en razón de incluir variables instrumentales (Inst), para el análisis, en el que todas las variables exógenas permiten explicar la endogeneidad de las variables del sistema de ecuaciones que forman el modelo. La producción científica del periodo anterior Producc $_{c i e}(-1)$ identifica la producción científica que aparece con tiempo rezagado, según Griliches (1998).

\section{RESULTADOS Y DISCUSIÓN}

Con una muestra pequeña de 12 observaciones, para un total de 96 datos, representada en los indicadores de ciencia, tecnología e innovación, registrados para Boyacá, en el periodo 2003 a 2014, se estima el modelo multiecuacional (ecuaciones 4 al 7); los datos se procesan con el paquete econométrico Eviews V.9, cuyos resultados ampliados se observan en la tabla 2 , en el que la producción científica total (variable dependiente) se relaciona con la inversión en ACTI y la gestión realizada por los grupos de investigación activos en relación con el número de graduados efectivos (variables explicativas), y otros factores no observables en el modelo, representados por $\varepsilon_{\mathrm{t} \text {. }}$

Tabla 2: Estimación del sistema de ecuaciones estructurales econométricas. Método: regresión aparentemente no relacionada

\begin{tabular}{|c|c|c|c|}
\hline Parámetros & Coefficient & $t$-Statistic & Prob. \\
\hline C (1) & -21.94737 & -8.012076 & 0.0000 \\
\hline C (2) & 1.777954 & 7.028597 & 0.0000 \\
\hline C (3) & 0.207153 & 1.022431 & 0.3157 \\
\hline C (4) & -3031.507 & -0.768659 & 0.4488 \\
\hline C (5) & 4005.850 & 3.689314 & 0.0010 \\
\hline C (6) & 276.8004 & 1.360874 & 0.1848 \\
\hline C (7) & -0.292341 & -0.881408 & 0.3859 \\
\hline C (8) & $-8.81 \mathrm{E}-07$ & -1.323273 & 0.1968 \\
\hline C (9) & 0.002348 & 3.435195 & 0.0019 \\
\hline
\end{tabular}

$\beta_{0}$, representa la tecnología; $\beta 1$, el efecto que ha tenido la inversión en ciencia, tecnología e innovación, y $\beta 2$, el efecto que los grupos de investigación activos y los graduados inmersos en los puestos de trabajo en las empresas ejercen sobre la producción científica. A priori, se espera que la relación entre grupos activos por 
número de graduados y la inversión en actividades científicas y de tecnología e innovación ACTI sea directamente proporcional a la producción científica.

Tabla 3: Test de normalidad en los residuales - Ortogonalización de Cholesky

\begin{tabular}{|c|c|c|c|}
\hline Component & Jarque-Bera & Df & Prob. \\
\hline 1 & 0.246949 & 2 & 0.8838 \\
\hline 2 & 0.332375 & 2 & 0.8469 \\
\hline 3 & 0.084426 & 2 & 0.9587 \\
\hline Joint & 0.663750 & 6 & 0.9952 \\
\hline
\end{tabular}

Dada la estimación de los parámetros $C(1)=\beta_{\circ}, C(2)=\beta 1, \ldots, C(9)=\beta 9$, se obtienen las siguientes ecuaciones:

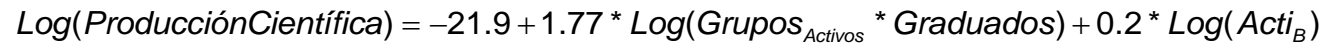

$$
\begin{aligned}
& \text { Valores } P t \text {-Statisc }=(0.0000) \quad(0.0000)(0.3157) \\
& R^{2}=0.88 \text { Durbin }- \text { Watson stat }=1.357335 \text { Prob. Jarque }- \text { Bera }=0.8838
\end{aligned}
$$

Examinando el cumplimiento de los supuestos del modelo, los errores o perturbaciones se distribuyen con probabilidad normal, dado que los valores de probabilidad $P$ de significancia del estadístico Jarque Bera son mayores a 0.05 , siendo este el nivel de significancia con el que se comparan todos los test aquí descritos (Ver tabla 3. test de normalidad). Así mismo se cumple la no multicolinealidad dado que los índices de condición son menores que 10. Además, en la ecuación (6), el valor del estadístico de Durbin Watson es de 2.33: está cercano a 2, indicando la no autocorrelación en los residuos, mientras que para las dos restantes ecuaciones, el estadístico de Durbin Watson, 1.35, no garantiza la no autocorrelación, motivo por el cual el modelo se estima por el método robusto Regresión aparentemente no relacionada, el cual corrige la autocorrelación. Los coeficientes de determinación alto $\left(R^{2}=88 \%, 58 \%\right.$ y $\left.59 \%\right)$ presentan buenos ajustes al modelo, lo cual indica que con estos porcentajes, la variabilidad de las variables predeterminadas explica a las variables endógenas en el modelo.

Si se observan los coeficientes de las variables exógenas en la ecuación (8), con la cual se explica la producción científica, en términos de publicación, los resultados muestran una elasticidad de 1.7 de la producción científica con respecto a los grupos de investigación activos y los graduados, indicando que al aumentar en un $1 \%$ la eficiencia del trabajo, medida por la anterior variable, las publicaciones aumentarán en $1.7 \%$, lo cual es significativo, si se observa el $p$, valor del estadístico $t=0<0.05$, nivel de significancia al cual se compara, y manteniendo constantes los demás factores. El signo positivo es el esperado, ya que según la teoría de Mankiw, a medida que mejora la tecnología existente, la eficiencia del trabajo aumenta; los aumentos de eficiencia de los graduados (E) producen el mismo efecto que el aumento de la población activa (L) en investigación. Es decir, la eficiencia en la interacción entre los grupos de investigación y los graduados inmersos en las organizaciones, es directamente proporcional a su producción científica. En términos de elasticidad, se confirma la teoría de Romer, quien considera que el conocimiento es otro factor de producción con retornos crecientes a escala. El análisis lo fundamenta en los procesos de generación de nuevos conocimientos -spillovers-, lo cual produce externalidades positivas que, a su vez, generan el crecimiento sin límite. En este sentido, se estima que los rendimientos, en el modelo tipo Coob-Douglas, son mayores a 1.

La inversión en las Actividades de Ciencia, Tecnología e Innovación (ACTI), para la producción científica (artículos publicados) no es significativa estadísticamente (el P-valor estadístico $t=0.31>0.05$ ). El signo positivo es el esperado, ya que la inversión es directamente proporcional a la producción, y cuando se trata de inversión en ACTI se justifica en los esfuerzos económicos y en otra serie de actividades importantes que no se reportan, pero que contribuyen a la investigación y desarrollo científico y tecnológico. Así, se refuta, en este caso, la tesis de Romer (1990) de que para producir conocimiento en I+D se requiere, aparentemente, de escasos recursos; por el contrario, se requiere de un volumen alto de inversión en ACTI para poder producir conocimiento, lo que lleva a ver con preocupación que la participación del PIB en inversión ACTI para Boyacá estuvo por debajo $0.11 \%$, en promedio. Así mismo, a priori, se espera que la relación entre los proyectos presentados a Colciencias y las becas otorgadas a los investigadores de doctorado sean directamente proporcionales a la inversión que se da en ACTI.

$$
\begin{aligned}
& \left(\text { Acti }_{b}\right)=-3031.5+4005.85^{*}\left(\text { Proyec }_{\text {colc }}\right)+276.8^{*}(\text { Becas }) \\
& \text { Valores } P t-\text { Statisc }=(0.4488)(0.0010)(0.1848) \\
& R^{2}=0.579511 \text { Durbin }- \text { Watson stat }=1.616436
\end{aligned}
$$


La ecuación (9) explica la relación que tiene el capital relacional, seleccionando como muestra los indicadores Proyectos financiados por Colciencias y Becas otorgadas, con la inversión en las ACTI para Boyacá. Al aprobarse un proyecto más en Colciencias, la inversión en ACTI para Boyacá será de 4005.85 (millones de pesos constantes de 2014). Esta variable es significativa estadísticamente (valor $P$ sig=0.001 $<0.05$, nivel de significancia con el cual se compara). El signo positivo es el esperado ya que el número de proyectos presentados es directamente proporcional a los recursos invertidos. Mientras que, las becas para investigación, no son significativa estadísticamente (valor P sig. $=0.18>0.05$, nivel de significancia con el cual se compara), en inversión en ACTI, manteniendo los demás factores constantes. Dado que los beneficios en términos de producción científica tienen efecto a largo plazo, la formación de capital humano no se alcanzó a visualizar con esta inversión.

Siguiendo con el análisis, el papel del conocimiento en los proyectos de investigación incentiva los beneficios futuros, y en esto es ilustrativo Marulanda,et al. (2013). De igual manera, a priori, se espera que, tanto el número de los graduados en el sistema de educación inmersos en las empresas y el número de grupos de investigación activos, así como la oferta de programas educativos de las universidades, sean directamente proporcionales a la creación de patentes. La ecuación (10) explica el comportamiento del capital estructural, seleccionando como muestra los indicadores Eficiencia del trabajo, Graduados, grupos de investigación activos y Oferta de programas en las universidades.

$$
\begin{aligned}
& \left.(\text { patentes })=-0.29-8.81 E-07^{*}\left(\text { Graduados }^{*} \text { Grupo }_{a c t i}\right)+0.002 * \text { Oferta }_{\text {programa }}\right) \\
& \text { Valores } P t-\text { Statisc }=(0.3859) \quad(0.1968)(0.0019) \\
& R^{2}=0.598040 \quad \text { Durbin }- \text { Watson stat }=2.336272
\end{aligned}
$$

Por cada nuevo programa ofertado por las universidades, la producción en patentes aumentaría en 0,002, constituyéndose en variable significativa (el $P$-valor es de 0,0019: menor que 0.05, valor con el que se compara), manteniendo los demás factores constantes. El signo positivo es el que se esperaba, teniendo en cuenta que en Boyacá, las estadísticas identifican un aumento en el número de programas, ofertados en todos los niveles académicos, con un efecto positivo en las innovaciones. Mientras que ante una variación unitaria en la eficiencia del trabajo dada por los grupos de investigación activos y los graduados, muestra una disminución en la concesión de patentes, no significativa al 5\% (el $P$ valor, que es de 0,1968, es mayor que 0.05), manteniendo los demás factores constantes. Teniendo en cuenta los datos reportados por el boletín Línea base de indicadores de Ciencia, Tecnología e Innovación para Boyacá, 2014 y 2015, en la mayoría de los períodos, el número de patentes registradas fue cero; uno, en algunos períodos, y dos patentes, como máximo; por este motivo, el signo se invierte, provocando una infinitesimal disminución, no representativa. Parece que no necesariamente el que las empresas no soliciten patentes implica que no haya innovación; puede haber innovación incremental en los procesos productivos o en los productos existentes, o simplemente se lanzan nuevos productos al mercado sin el uso de patentes, lo que coincide con el hecho de que tener una proporción grande de micro y pequeñas empresas suele distorsionar el indicador, debido a que una parte considerable de ellas efectúan algún tipo de gasto en I+D que nunca se registra, el cual, la mayoría de las veces, es menor al de otras actividades de investigación poco significativas, coincidiendo con los argumentos tanto de Ríos y Ocegueda (2017) como los de (Neumann y Weigand, 2004; Callón, (1999).

\section{DISCUSION FINAL}

Analizados los diferentes escenarios, se verifica que con la inversión destinada a las actividades de ciencia, tecnología e innovación —reflejada en las publicaciones de calidad (registradas en WoS) - y con la interacción entre los grupos de investigación y la educación formal e informal existente en el sistema educativo, se incrementa la producción científica a mayor escala, principalmente, a través de los conocimientos y habilidades adquiridos, relacionados con el capital humano. Lo anterior permite generar ventajas competitivas que incentivan la producción científica en el departamento de Boyacá. Por su parte, el capital relacional en ciencia, tecnología e innovación para Boyacá, está representado significativamente en los proyectos que se desarrollan en conjunto con Colciencias, así como en la importante contribución que tiene la asignación eficiente de los recursos en las becas, en especial para doctorado, las cuales devienen en beneficios en las tasas de inversión, manteniendo criterios de rentabilidad social.

Si se evalúa la producción científica al combinar los esfuerzos entre universidades, Estado y empresa, en términos de la oferta de programas de las instituciones educativas y los proyectos avalados por Colciencias, así como de las becas otorgadas a investigadores, se puede comprobar que crecerá la producción científica. Adicionalmente, la activación del capital relacional de las empresas con las universidades y el Estado fomenta la competitividad en un entorno de continuos cambios, y responde dinámicamente a las preferencias, necesidades y tecnologías presentes, impulsando la habilidad de los trabajadores para cambiar e innovar rápidamente. 
La obtención de patentes es resultado de la eficiencia en gestión que los grupos de investigación activos generan, las acciones que cada uno de los graduados puede haber aportado en los puestos de trabajo, la oferta de programas de las universidades en los distintos niveles profesionales y el conocimiento provisto por dichos programas, que fortalecen las capacidades de innovación de las empresas, a futuro.

Se evidencian, pues, los retos que aún enfrenta la estructura productiva del sector manufacturero en Colombia, y que no son diferentes para Boyacá, cuyas deficiencias en materia de innovación en sectores estratégicos no permiten generar cambio técnico y el consecuente mejoramiento de sus productos. Respecto al sector servicios, al igual que el uso de registros industriales - que incluso puede ser más representativo de la innovación que el uso de las patentes y los modelos de utilidad-, los registros de software también son bajos en este sector.

\section{CONCLUSIONES}

De acuerdo al trabajo presentado y a los resultados obtenidos, se pueden plantear las siguientes conclusiones principales:

1.- La teoría económica y administrativa y el análisis econométrico permiten comprender la gestión del conocimiento en CTel como una relación entre el capital humano, el capital estructural y el capital relacional, denominado capital intelectual. Este está representado en las personas, la infraestructura y el uso de la tecnología, la información y el conocimiento, elementos que promueven las innovaciones y el reconocimiento de la sociedad; clave para la competitividad y el desarrollo en las diferentes entidades educativas y las organizaciones en general.

2.- Es ilustrativo encontrar que el capital humano impulse al capital estructural y al capital relacional, en las organizaciones. Sin embargo, para que sea significativa la producción científica se requiere de mayor inversión económica.

3.- El registro de los proyectos de investigación en Colciencias permite mejorar la distribución y radio de acción de los recursos invertidos, y visualizar la gestión en CTel de una región. A partir de ello, analiza sus alcances y limitaciones, al tiempo que hace seguimiento a los desarrollos estructurales dentro de las organizaciones.

\section{AGRADECIMIENTOS}

A la Dirección de Investigación de la UPTC por la financiación del proyecto Análisis Estadístico de Los Indicadores de Ciencia, Tecnología e Innovación (CTel) de Boyacá, Colombia, _desarrollado en 2016 - 2017 y ejecutado por el grupo de Investigación en Estadística GAMMA de la UPTC_; A Judy Jannely Pérez Ramírez, estudiante de Economía de la UPTC y miembro del semillero del grupo de investigación GAMMA, por su constante apoyo en la logística y parte del estudio.

\section{REFERENCIAS}

Ahuja, G., Collaboration Networks, Structural Holes, and Innovation: A Longitudinal Study, DOI: 10.2307/2667105, Administrative Science Quarterly, 45(3), 425-455 (2000)

Álvarez, E., y C. Bernal, Modelo de Innovación Abierta: Énfasis en el Potencial Humano, doi.org/10.4067/S071807642017000100007, Información Tecnológica, 28 (1), 65-76 (2017)

Arrow, K., The Economic Implication of Learning by Doing, DOI: 10.2307/2295952, Review of Economic Studies, 29 (3), 155-173 (1962)

Becheikh, N., R. Landry y N. Amara, Lessons from Innovation Empirical Studies in the Manufacturing Sector: A Systematic Review of the Literature from 1993-2003, DOI:10.1016/j.technovation.2005.06.016, Technovation, 26 (5), $644-664$ (2006)

Becker, G. S., Human Capital: A Theoretical and Empirical Analysis, with Special Reference to Education, 2a Ed., 1-264, Economic Research, New York (1975)

Bueno, E., M. Salvador y C. Merino, Génesis, Concepto y Desarrollo del Capital Intelectual en la Economía del Conocimiento: una reflexión sobre el modelo intellectus y sus aplicaciones, Estudios de Economía Aplicada, ISSN: 11333197, 26 (2), 43-63 (2008)

Callón, M., The Role of Lay People in the Production and Dissemination of Scientific Knowledge, doi.org/10.1177/097172189900400106, Science technology \& society, 4 (1), 81-94 (1999)

Crepon, B., E. Duguet y J. Mairessec, Research, Innovation And Productivi[Ty: An Econometric Analysis At The Firm Leve. doi.org/10.1080/10438599800000031, Economics of Innovation and New Technology, 7 (2), 115-158 (1998)

Cruz, V.A., Sistema de Gestión de la Calidad en el Apoyo a la Implementación de Estrategias de Producción Ajustada, 
doi.org/10.4067/S0718-07642004000600010, Información Tecnológica, 15 (6), 63-70 (2004)

DANE, Boletín Departamento Administrativo Nacional de Estadísticas, DANE, Bogotá, Colombia (2013)

Díez, M., L. Ochoa y A. Santidrián, Intellectual Capital and Value Creation in Spanish Firms, DOI: 10.1108/14691931011064581, Journal of Intellectual Capital, 11 (3), 348-367 (2010)

Echeverri, A., N. Lozada y J. Arias, Incidencia de las Prácticas de Gestión del Conocimiento sobre la Creatividad Organizacional. doi.org/10.4067/S0718-07642018000100071, Información Tecnológica, 29(1), 71-82 (2018)

Espejo, R.L., Interacción Simbólica en un Acto de Subjetividad de Género, doi: 10.4067/S0718-50062015000500006, Formación Universitaria, 8(5), 43-58 (2015)

Espejo, R. L., Subjetividades Generizadas en Formadores y Formadoras. Experiencias de Licenciados/as en Educación, 1르. Ed., 31-36, ISBN: 978-958-660-265-5, Editorial UPTC, Tunja, Colombia (2018)

Fagerberg, J., S. Martin y V. Bart, Innovation and Economic Developmen, doi.org/10.1016/S0169-7218(10)02004-6, Handbook of Economics of Innovation, 2, 833-872 (2010)

Frietsch, R., I. Haller, M. Funken-Vrohlings y H. Grupp, Gender-specific Patterns in Patenting and Publishing, doi.org/10.1016/j.respol.2009.01.019, Research policy, 38 (4), 590-599 (2009)

Griliches, Z., R \& D and Productivity: the Econometric Evidence, 19-138, ISBN-0226-30886-3, The University of Chicago Press, United States of America (1998)

Gujarati, D., Econometría. 5ª Ed., 673-773, ISBN: 978-607-15-0294-0, McGraw Hill, Bogotá, Colombia (2010)

Guzmán, A., F., López y F. Venegas, Un Análisis de Cointegración entre Patentes y Crecimiento Económico en México, 1980-2008, Investigación Económica, 71 (281), 83-115 (2012)

Hernández, H., D. Cardona y J. Del Rio, Direccionamiento Estratégico: Proyección de la Innovación Tecnológica y Gestión Administrativa en las Pequeñas Empresas, doi:10.4067/S0718-07642017000500003, Información Tecnológica, 28(5), 1522 (2017)

Hernández, A., C. Marulanda y M. López, Análisis de Capacidades de Gestión del Conocimiento para la Competitividad de Pymes en Colombia, doi.org/10.4067/S0718-07642014000200013, Información Tecnológica, 25 (2), 111-122 (2014)

Hernández, N., C. Fernández y P. Baptista, Metodología de la investigación, ISBN: 970-10-5753-8, McGraw-Hill Interamericana, México (2006)

Hitt, M., J. Gimeno, y R. Hoskisson, Current and Future Research Methods in Strategic Management, doi.org/10.1177/109442819800100103, Organizational Research Methods, 1 (1), 6-44 (1998)

Información Tecnológica: 25 años Difundiendo la Ciencia y la Tecnología Iberoamericana, doi.org/10.4067/S071807642014000600001, Información Tecnológica, 25 (6), 1-2 (2014)

Langebaek, A. y D. Escobar, Determinantes de la Actividad Innovadora en la Industria Manufacturera Colombiana, Borradores de Economía, (433), 5-35 (2007)

Link, A. y D. Siegel, Generating science-based growth: an econometric analysis of the impact of organizational incentives on university-industry technology transfer, DOI: 10.1080/1351847042000254211, The European Journal of Finance, 11 (3), 169 -181 (2005)

López, D., C. Marulanda y M. López, Métricas de Valoración de la Gestión del Conocimiento para las Pequeñas y Medianas Empresas del Sector Tecnologías de Información en el Triángulo del Café en Colombia. doi:org/10.4067/S071807642015000300020, Información Tecnológica, 26(3), 173-183 (2015)

Lucas, R., On the Mechanics of Economic Development, Journal of Monetary Economics, 22, 3-42 (1998)

Luintel, K. y K. Mosahid, Heterogeneous Ideas Production, DOI: 10.1111/j.1540-5982.2009.01543, Canadian Journal of economics Revue Cannadiene deconomique, 42 (3), 1176-1205 (2009)

Mairesse, J. y P., Mohnen, Chapter 26 - Using Innovation Surveys for Econometric Analysis, doi.org/10.1016/S01697218(10)02010-1, ISBN: 978-0-444-53609-9, Handbook of the Economics of Innovation, 2, 733-1256 (2010)

Mankiw, G., Macroeconomía, 4 Ed., 1-701, ISBN: 958-97647-6-2, Editorial Anthony Bosch, Barcelona, España (2005)

Martínez, M., De la Contabilidad de los Recursos Humanos al Capital Intelectual y la Gestión del Conocimiento: una Ampliación Necesaria. Dirección y Organización, ISSN: 1132-175X, Revista de dirección, organización y administración de empresas, 29, 134-144 (2003)

Marulanda, C., J. Giraldo y M. López, Evaluación de la Gestión del Conocimiento en las Organizaciones de la Red de Tecnologías de Información y Comunicaciones del Eje Cafetero en Colombia, doi:org/10.4067/S071807642013000400012, Información Tecnológica, 24(4), 105-116 (2013)

Mateus, S. y J., Giraldo, Diseño de un Modelo 3D del Politécnico Colombiano Jaime Isaza Cadavid con Realidad Virtual, doi.org/10.4067/S0718-07642012000300012, Información Tecnológica, 23 (3), 95-102 (2012)

Neumann M. y J. Weigand (Eds.), The International Handbook of Competition, ISBN: 978-I- 84376-054-2 (cased), Edward Publishing (EE). Massachusetts-USA, 339 (2004) 
Palomo, I., C. Veloso y R. Schmal, Sistema de Gestión de la Investigación en la Universidad de Talca, doi.org/10.4067/S0718-07642007000100014, Información Tecnológica, 18 (1), 97-106 (2007)

Pinto, S., P. Ramírez y E. Grandón, Antecedentes del Éxito de los Sistemas de Planificación de Recursos Empresariales en las Grandes Empresas Chilenas: un Modelo Factorial Exploratorio, doi.org/10.4067/S0718-07642017000300015, Información Tecnológica, 28(3), 139-146 (2017)

Pires, A. y V., Machado, Gestión por Procesos en el Diseño de las Organizaciones, doi.org/10.4067/S071807642006000100005, Información Tecnológica, 17 (1), 35-44 (2006)

Quiroga D., B. Hernández, J. Torrent y J. Ramírez, La Innovación de Productos en las Empresas Caso Empresa América Latina, Cuadernos del Cendes, 31(87), 63-85 (2014)

Ríos, J. y J. Ocegueda, Capacidad Innovadora y el Desempeño Económico y Tecnológico en México, DOI: 10.22136/est2017705, Economía sociedad y territorio, XVII (55), 743-776 (2017)

Rodríguez, A., A. Araujo y J. Urrutia, La Gestión del Conocimiento Científico-Técnico en la Universidad: un Caso y un Proyecto, Cuadernos de Gestión, 1 (1), 13-30 (2001)

Romer, P., Endogenous Technological Change, Journal of Political, 98 (5) 71-102 (1990)

Romer, P., The Origins of Endogenous Growth, DOI: 10.1257/jep.8.1.3, The Journal of Economic Perspectives, 8 (1) 3-22 (1994)

Sancho, R., Indicadores de los Sistemas de Ciencia, Tecnología e Innovación, Economía Industrial, 343 (I) 97-109 (2002)

Santos, H., P. Figueroa y C. Fernandez, The Influence of Human Capital on the Innovativeness of Firms, The International Business \& Economics, DOI https://doi.org/10.19030/iber.v9i9.625, Research Journal, 9(9), 53-64 (2010)

Schultz, T., Investing in People: The Economics of Population Quality, ISBN: 0-478-4787-7, University of California press, Los Angeles, London, 167 (1981)

Schumpeter, J., Teoría del Desenvolvimiento Económico. Una investigación sobre Ganancias, Capital, Crédito, Interés y Ciclo Económico. $2^{\circ}$ Ed., 256, ISBN: 9681602090, Fondo de Cultura Económica, México (1997)

Secundo, G., A. Margherita, G. Elia y G. Passiante, Intangible Assets in Higher Education and Research: Mission, Performance or Both? DOI: 10.1108/14691931011039651, Journal of Intellectual Capital, 11 (2), 140-157 (2010)

Tejedor, R., J. Gil y J. Tejedor, Derechos de Propiedad Intelectual (DPI) y Crecimiento Económico: Una Revisión, doi.org/10.18359/rfce.3145, Rev. Fac. Cienc. Econ., XXVI (1), 225-243 (2018)

Universidad Pedagógica y Tecnológica de Colombia Boletín de Indicadores de Ciencia, Tecnología e Innovación para Boyacá, DIN, Tunja, Boyacá, Colombia (2014)

Universidad Pedagógica y Tecnológica de Colombia Boletín de Indicadores de Ciencia, Tecnología e Innovación para Boyacá, DIN, Tunja, Boyacá, Colombia (2015)

Valdés P., R. Valdés, J. Guisasola y T. Santos., Implicaciones de las Relaciones Ciencia-Tecnología en la Educación Científica, Revista IBERO AMERICANA de Educación, 28, 101-128 (2002)

Wolfgang, B. y D. Jürgen, R\&D, Cooperation and Innovation Activities of Firms: Evidence for the German Manufacturing Industry, Research Policy, 33 (2), 209-223 (2002) 